\title{
ON A SYSTEM OF INTEGRAL INEQUALITIES ${ }^{1}$
}

\author{
S. G. DEO ${ }^{2}$ AND M. G. MURDESHWAR
}

Abstract. The present note obtains a vector extension and a further generalization of Bihari's Lemma on an integral inequality. The inequality proved can be used in the study of the componentwise behaviour of solutions of differential systems.

The theory of integral inequalities plays a vital role in the study of the stability properties of differential and integral equations. In particular, Bihari's integral inequality continues to be an effective tool to study sophisticated problems such as stability, boundedness and uniqueness of solutions [2], [3]. The statement of this lemma is as follows:

LEMMA (BIHARI). (i) Let $x$ and $F$ be nonnegative continuous functions on $[0, \alpha)$, and

(ii) $\omega$ a positive nondecreasing continuous function on $(0, \infty)$ such that

$$
x(t) \leqq k+M \int_{0}^{t} F(s) \omega(x(s)) d s, \quad t \in[0, \alpha),
$$

where $k$ and $M$ are positive constants. Then

$$
x(t) \leqq G^{-1}\left(G(k)+M \int_{0}^{t} F(s) d s\right), \quad t \in\left[0, \alpha_{1}\right),
$$

where

$$
G(u)=\int_{u_{0}}^{u} \frac{d t}{\omega(t)}, \quad u \geqq u_{0}>0,
$$

and $G^{-1}$ is the inverse function of $G$ and

$$
\alpha_{1}=\sup \left[t \in[0, \alpha): G(\infty) \geqq G(k)+M \int_{0}^{t} F(s) d s\right] .
$$

In this note we shall present (i) an extension to systems and (ii) a further generalization of the above lemma so that it will be effective in more delicate situations.

Received by the editors January 28, 1970.

AMS 1969 subject classifications. Primary 3490; Secondary 3442.

Key words and phrases. Integral inequality, Bihari's Lemma.

1 This work was supported by NRC Canada Operating Grants A-3072 and A-3074.

2 This author is on leave from Marathwada University, Aurangabad. India. 
Let $R^{n}$ denote the $n$-dimensional Euclidean space and $x_{1}, x_{2}, \cdots, x_{n}$ are the components of an element $x \in R^{n}$. Assume that, whenever the subscript $i$ occurs, it takes the values from 1 to $n$. Suppose that $\omega \in C\left[R^{n}, R^{n}\right]$ and the functions $\omega_{i}\left(u_{1}, u_{2}, \cdots, u_{n}\right)>0$ are nondecreasing in $u_{1}, u_{2}, \cdots, u_{n}$. Assume that $\lim \omega_{i}\left(u_{1}, u_{2}, \cdots, u_{i}, \cdots, u_{n}\right)$, as each component except $u_{i}$ tends to $\infty$, exists and is denoted by $\tilde{\omega}_{i}\left(u_{i}\right)$. Now we prove the following theorem.

THEOREM 1. If

(i) $x_{i}, F_{i}$ are nonnegative continuous functions on $[0, \alpha)$, and

(ii) the vector functions $\omega$ and $\tilde{\omega}$, as described above, are such that

$$
x_{i}(t) \leqq k_{i}+M_{i} \int_{0}^{t} F_{i}(s) \omega_{i}(x(s)) d s, \quad t \in[0, \alpha),
$$

where $k_{i}$ and $M_{i}$ are positive constants, then

$$
x_{i}(t) \leqq G_{i}^{-1}\left(G_{i}\left(k_{i}\right)+M_{i} \int_{0}^{t} F_{i}(s) d s\right), \quad t \in[0, \bar{\alpha}),
$$

where

$$
G_{i}\left(u_{i}\right)=\int_{u_{i 0}}^{u_{i}} \frac{d y_{i}}{\tilde{\omega}_{i}\left(y_{i}\right)}, \quad u_{i} \geqq u_{i 0}>0,
$$

and $G_{i}^{-1}$ is the inverse function of $G_{i}$ and $[0, \bar{\alpha})=\min _{i}\left[0, \alpha_{i}\right)$ where

$$
\alpha_{i}=\sup \left[t \in[0, \alpha): G_{i}(\infty) \geqq G_{i}\left(k_{i}\right)+M_{i} \int_{0}^{t} F_{i}(s) d s\right] .
$$

Proof. Let the right-hand side of (1) be denoted by $z_{i}(t)$. Then

$$
z_{i}^{\prime}(t)=M_{i} F_{i}(t) \omega_{i}(x(t)) \leqq M_{i} F_{i}(t) \tilde{\omega}_{i}\left(z_{i}(t)\right)
$$

which in view of (3) reduces to

$$
d G_{i}\left(z_{i}\right) / d t \leqq M_{i} F_{i}(t) .
$$

Now the conclusion (2) follows on integrating (4) from 0 to $t$. The domain of the function $G^{-1}$ is obvious (cf. [3]).

REMARK 1. It is easy to prove the following inequality. Let the assumptions (i) and (ii) of Theorem 1 hold. If the inequality (1) is replaced by

$$
x_{i}(t) \geqq k_{i}-M_{i} \int_{0}^{t} F_{i}(s) \omega_{i}(x(s)) d s, \quad t \in[0, \alpha),
$$

then 


$$
x_{i}(t) \geqq G_{i}^{-1}\left[G_{i}\left(k_{i}\right)-M_{i} \int_{0}^{t} F_{i}(s) d s\right]
$$

on $[0, \tilde{\alpha})=\min _{i}\left[0, \alpha_{i}\right)$, where

$$
\alpha_{i}=\sup \left[t \in[0, \alpha): G_{i}\left(k_{i}\right)>M_{i} \int_{0}^{t} F_{i}(s) d s\right] .
$$

This extends the inequality proved in [4]. Now we obtain a generalization of the same lemma.

THEOREM 2. Let the assumptions (i) and (ii) of Theorem 1 hold and $f$ be a strictly increasing and continuous function on $[0, \infty)$ such that $f(0)=u_{i 0}, f(\infty)=\infty$, and

$$
f\left(x_{i}(t)\right) \leqq k_{i}+M_{i} \int_{0}^{t} F_{i}(s) \omega_{i}(x(s)) d s, \quad t \in[0, \alpha),
$$

where $k_{i}$ and $M_{i}$ are positive constants. Then

$$
f\left(x_{i}(t)\right) \leqq G_{i}^{-1}\left(G_{i}\left(k_{i}\right)+M_{i} \int_{0}^{t} F_{i}(s) d s\right), \quad t \in[0, \bar{\alpha}),
$$

where

$$
G_{i}\left(u_{i}\right)=\int_{u_{i 0}}^{u_{i}} \frac{d y_{i}}{\tilde{\omega}_{i}\left(f^{-1}\left(y_{i}\right)\right)}, \quad u_{i} \geqq u_{i 0}>0,
$$

and $G_{i}^{-1}$ is the inverse function of $G_{i}$ and $[0, \bar{\alpha})=\min _{i}\left[0, \alpha_{i}\right)$ where

$$
\alpha_{i}=\sup \left[t \in[0, \alpha): G_{i}(\infty) \geqq G_{i}\left(k_{i}\right)+M_{i} \int_{0}^{t} F_{i}(s) d s\right] .
$$

Proof. Let the right-hand side of (5) be denoted by $z_{i}(t)$. Then, it is easy to observe that

$$
M_{i} F_{i}(t) \omega_{i}(x(t)) \leqq M_{i} F_{i}(t) \tilde{\omega}_{i}\left(f^{-1}\left(z_{i}(t)\right)\right)
$$

which in view of (7) can be written as

$$
d G_{i}\left(z_{i}\right) / d t \leqq M_{i} F_{i}(t) .
$$

The conclusion (6) is now clear.

REMARK 2. The inequality (5) is much stronger than the inequality (1). In fact, if $f^{-1}=g$ and the function $g$ is subadditive then the inequality (5) takes the form

$$
x_{i}(t) \leqq \eta_{i}+g\left(M_{i} \int_{0}^{t} F_{i}(s) \omega_{i}(x(s)) d s\right) .
$$


This inequality is much more general and it includes the inequalities proved in [1], [2] as special cases.

REMARK 3. It has been established in [3] that the comparison method provides a powerful tool for obtaining bounds for solutions and it is a unified approach to many similar problems. The comparison equation employed in [2], [3] is a scalar one. It is now possible to extend these results where a scalar equation can be replaced by a system. This approach will facilitate the study of the componentwise behaviour of a solution of a differential system. The details are omitted.

\section{REFERENCES}

1. R. Bellman, The stability of solutions of linear differential equations, Duke Math. J. 10 (1943), 643-647. MR 5, 145.

2. I. Bihari, $A$ generalization of a lemma of Bellman and its application to uniqueness problems of differential equations, Acta. Math. Acad. Sci. Hungar. 7 (1956), 81-94. MR 18, 38.

3. F. Brauer, Bounds for solutions of ordinary differential equations, Proc. Amer. Math. Soc. 14 (1963), 36-43. MR 26 \#397.

4. C. E. LANGENHOP, Bounds on the norm of a solution of a general differential equation, Proc. Amer. Math. Soc. 11 (1960), 795-799. MR 22 \#12260.

University of Alberta, Edmonton, Alberta, Canada 